\title{
A Novel Main-Lobe Cancellation Method Based on a Single Notch Space Filter and Optimized Correlation Analysis Strategy
}

\author{
Jiazhi Zhang $\mathbb{D}^{1}{ }^{1}$ Xin Zhang $\mathbb{D}^{1,2}$ Weibo Deng, ${ }^{1,2}$ Liang Guo, ${ }^{1}$ and Qiang Yang $\mathbb{D}^{1,2}$ \\ ${ }^{1}$ School of Electronics Information Engineering, Harbin Institute of Technology, Harbin 150001, China \\ ${ }^{2}$ Collaborative Innovation Centre of Information Sensing and Understanding at Harbin Institute of Technology, \\ Harbin 150001, China \\ Correspondence should be addressed to Qiang Yang; yq@hit.edu.cn
}

Received 16 July 2018; Revised 30 October 2018; Accepted 21 November 2018; Published 7 March 2019

Academic Editor: Atsushi Mase

Copyright (c) 2019 Jiazhi Zhang et al. This is an open access article distributed under the Creative Commons Attribution License, which permits unrestricted use, distribution, and reproduction in any medium, provided the original work is properly cited.

The high-frequency hybrid sky-surface wave radar (HFSSWR) is a new kind of over-the-horizon radar system. Combining the advantages of both the sky wave radar and the surface wave radar, HFSSWR has drawn much attention in recent years. But the new system also brings new challenges. One of the most severe problems is that the heterogeneous environment makes a big challenge for the sea clutter suppression processing. Due to the nonstationary properties of the ionosphere, the first-order sea clutter statistics change significantly among range bins. So the new efficient sea clutter suppression method is required, and the clutter characteristics analyses are also in urgent need of research in order to guide the design of the algorithm in the background of HFSSWR. In this paper, utilizing the measured data set, we first analyse the range and spatial characteristics of the nonhomogeneous first-order sea clutter in HFSSWR. Then, an improved main-lobe cancellation (IMLC) method based on single notch space filter and correlation analysis is proposed to get training data which contains precise clutter information. A main-lobe clutter canceller based on the single notch space filter has been developed to block the target component, and an optimized correlation analysis (OCA) strategy is presented to choose the efficient training data. Finally, the method is examined by measured data and the results indicate that the method has a good performance for broadening first-order sea clutter suppression than the traditional method.

\section{Introduction}

High-frequency hybrid sky-surface wave radar is a novel radar system and has developed to a new direction in HF over-the-horizon radar (OTHR) areas. It is a natural combination of a sky wave transmitting propagation mode and a surface wave receiving propagation mode [1]. The geometric sketch map of the HFSSWR is shown in Figure 1. In recent years, it has attracted OTHR researchers' attention due to the mixed propagation mode system [2-6]. Theoretically, it can overcome the close-range blind zone and mitigate the effect of the strong ionospheric modulation in HF skywave radar as well as avoid the vertical ionospheric clutter in HF surface-wave radar (HFSWR). However, challenges have been brought in by the new radar configuration. One of the most severe problems is the broadened sea clutter contaminated by the ionosphere. In the case of ionosphere destabilization, the Bragg peaks of the first-order sea clutter broaden severely and have a complex shape. And the bistatic radar system also exacerbates the effect as the Bragg frequency changes with the bistatic and pitch angle in a cell.

The broadened first-order sea clutter which spreads in angle and Doppler dimension will submerge the lowvelocity vessels which travel with the radial velocity close to the Bragg lines [7]. In terms of the clutter suppression algorithm design, research on the clutter characteristics is required to give the breach of the problem. It is of vital importance to study the properties of the clutter which need to be suppressed and find the appropriate algorithm which is efficient to do the suppression. So in this paper, the characteristic analysis of the broadened first-order sea clutter of HFSSWR is studied for the first time utilizing the 


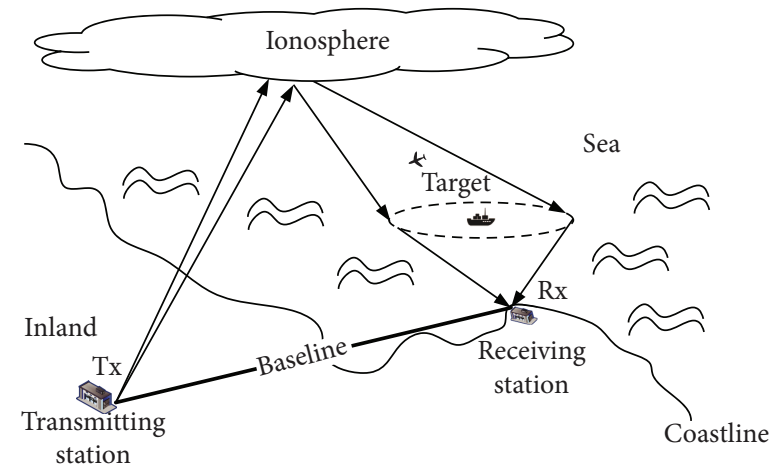

FIGURE 1: Sketch map of HFSSWR.

measured data. The range and spatial characteristics have been analysed to give the guidance of the clutter suppression algorithm design. The range property influences how to select the training data to estimate the covariance matrix of the cell under test and the spatial property effects on how to reject the target component. The main-lobe cancellation (MLC) method has been presented to suppress the sea clutter in HFSWR [8]. It achieves clutter suppression by obtaining target-free training data to estimate clutter information in the looking direction with the clutter in other directions. So the clutter in the cell under test (CUT) can be cancelled by the estimation, and the target signal can be protected at the same time. The training data is obtained from a large amount of range cell data which is required to be independent and identically distributed (iid) with the test cell data [9-11]. In HF surface wave radar, the sea clutter is rather stationary with fewer disturbances from the ionosphere. So it is easier to provide more valid training data in the range domain. But in heterogeneous environments, like HF shipborne radar and hybrid sky-surface wave radar, the clutter statistics change significantly due to the highly variable nature of radiowave propagation through the ionosphere [12], which makes the cancellation method invalid. So in order to apply the MLC method to suppress the sea clutter, the analysis of the clutter's range and spatial characteristics is needed and how to select valid training data is the key problem in the background of HFSSWR.

In this paper, we first analyse the characteristics of the nonhomogeneous first-order sea clutter in HFSSWR utilizing the measured data to prove the necessity of the training data selection method in Section 2. On this basis, in Section 3, we develop a main-lobe clutter canceller based on a single notch space filter to obtain the training data rejecting the target information. Then, an improved training data selection method, which we call the optimized correlation analysis strategy (OCA) based on the spatial eigenvector, is developed to suppress the broadened first-order sea clutter, shown in Section 4. Finally, the method is examined by measured data in Section 5 and the results indicate that the method has a good performance for broadening first-order sea clutter suppression than the traditional method. And the conclusion is presented in Section 6. It is noteworthy that the basic framework of the method is previously presented in [13], but there are several key problems that need to be discussed together with some new research progress as shown in this paper. The analyses of the impact of amplitude-phase errors are shown in Section 3.3, the simulation results of the broadening value of the first-order sea clutter are presented in Section 4.2 , and a new clutter suppression experiment with a noncooperative target is shown in Section 5.2.

\section{Characteristic Analysis of Broaden First-Order Sea Clutter}

The main-lobe cancellation method has been proposed to suppress the space spread clutter of HFSWR in [8]. The main idea of the method is to estimate the clutter in the looking direction utilizing the clutter in other directions, and the clutter can be cancelled by subtraction. In this case, the secondary data which is used to estimate the clutter information in other directions must be target-free. And in adaptive processing, the secondary data is usually collected from adjacent range cells. It utilizes two characteristics of clutter in HFSWR. One is that the sea clutter characteristic in the range dimension is stable so that there is a large amount of training data which can be seen as independent and identically distributed with the cell under test. The other is the different spatial characteristics of clutter and target. In HFSWR, the space spread clutter has no directional property; it occurs almost in every observation direction. On the contrary, the target has a strong direction, the echo signal of the target only receives from a certain area, so that the main-lobe clutter can be suppressed by the clutter in the other side-lobe and the target can be protected at the same time. However, in terms of the background of HFSSWR, there are few researches that have been done on these two characteristics. The analyses of these properties are needed if the powerful method is going to be applied in HFSSWR to deal with the clutter here.

So in Sections 2.1 and 2.2, we analyse the two clutter characteristics utilizing the measured data of HFSSWR. It is the foundation of the algorithm design.

The measured data is obtained through the HFSSWR system in Central and Eastern China on June 10, 2015, and then processed by matched filters in range, Doppler, and digital beam-forming in turn. Figure 2 gives a typical rangeDoppler spectrum.

We can see from Figure 2 that the clutter background is complicated for the practical HFSSWR system. There are typically 4 kinds of clutters which will have negative effects on target detection. First is the strong direct wave clutter in the close range area. Second is the spread ionospheric clutter in the far-range area which covers a wide range of range and Doppler bins. Fortunately, these two clutters usually occur in the range regions of no interest. But in the regions of interest for the HFSSWR system, there are two types of clutters which are the major problems for clutter suppression processing. The linear ionospheric clutter covers a few Doppler bins which appears unpredictable. And the broadening firstorder sea clutter badly affects low-velocity vessel detection if the vessels' velocities are close to the Bragg peaks. So in this section, we do research on the characteristics of the firstorder sea clutter in the background of HFSSWR. The 


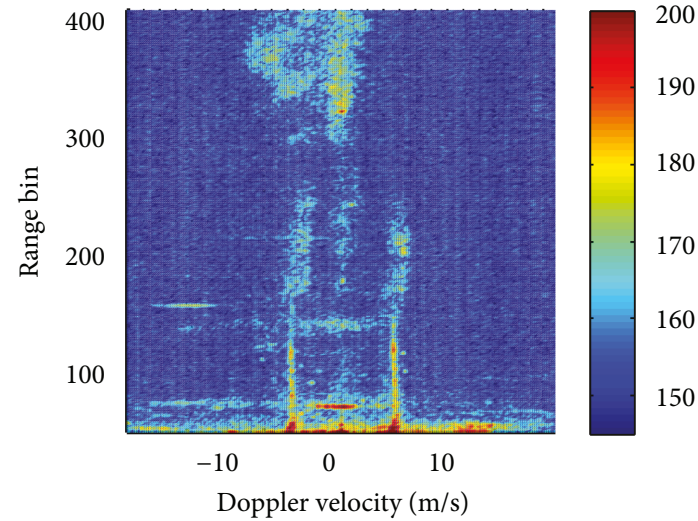

FIGURE 2: Range-Doppler spectrum of HFSSWR.

analyses of clutter characteristics will guide the design of the clutter suppression algorithm.

2.1. Range Characteristics of Sea Clutter. To analyse the clutter characteristics in the range domain, we first give a range spectrum of a negative Bragg peak to show the intuitionistic character in Figure 3.

We can see that the clutter signal power spectrum changes rapidly in the range dimension and it is hard to model the first-order sea clutter's power spectrum. The first several range bins are influenced by the strong direct wave. The spread ionospheric clutter occurs in the far region and covers a wide range bins as well as the Doppler bins (shown in Figure 2).

The correlation analysis is an effective way to show the signal characterization. In the range domain, the correlation coefficient of the data in different range bins can be calculated by

$$
\eta_{i, j}=\frac{\left(1 / N_{f_{\mathrm{d}}}\right) \sum_{N_{f_{\mathrm{d}}}} Z_{\mathrm{b}}\left(i, f_{\mathrm{d}}\right) Z_{\mathrm{b}}^{*}\left(j, f_{\mathrm{d}}\right)}{\sqrt{\left(1 / N_{f_{\mathrm{d}}}\right)^{2} \sum_{N_{f_{\mathrm{d}}}}\left|Z_{\mathrm{b}}\left(i, f_{\mathrm{d}}\right)\right|^{2} \sum_{N_{f_{\mathrm{d}}}}\left|Z_{\mathrm{b}}^{*}\left(j, f_{\mathrm{d}}\right)\right|^{2}}},
$$

where $N_{f_{\mathrm{d}}}$ is the number of the sample Doppler bin, $i$ is the reference range bin, $j=1,2, \ldots, R$ is the number of whole range bins which are going to be analysed, $Z_{\mathrm{b}}$ is the sample data in the beam bin $b$ with Doppler shift $f_{\mathrm{d}}$, and $(\cdot)^{*}$ represents the complex conjugate operation.

The results are shown in Figure 4. In Figure 4(a), the Doppler frequency is $f=-0.3255 \mathrm{~Hz}$, which is the negative Bragg peak, the beam direction is $b=-6^{\circ}$, and the reference range bin is $i=86$. We can see that the correlation coefficients in this situation are at a low level among the whole range bins except the reference range bin itself. This shows that the ionosphere's statistical property changes rapidly during the experiment time. The state of the first-order sea clutter is not stable at even the adjacent range bins due to the ionospheric disturbance. This highly nonstationary environment can be seen as a distinguishing feature for the sea clutter in hybrid sky-surface wave mode compared with the simple surface wave radar. In this case, there will be no training data available which is required for common adaptive filter

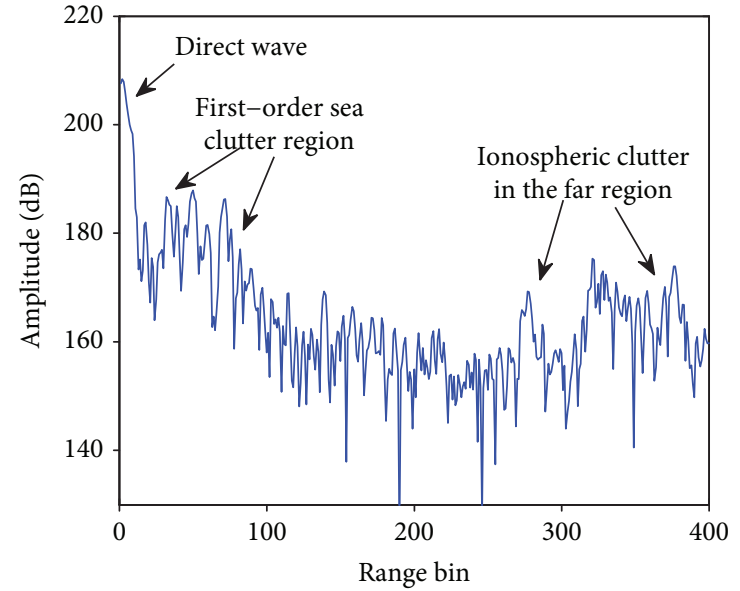

FIGURE 3: Range spectrum of the no. 130 Doppler bin.

methods. This sample starved scenario is called single dataset situation, and the related method is presented in [12].

In Figure 4(b), the Doppler frequency is $f=0.3516 \mathrm{~Hz}$, which is the positive Bragg peak, the beam direction is $b$ $=0^{\circ}$, and the reference range bin is $i=77$. The result differs from that shown in Figure 4(a). We can see that the correlation coefficients in this situation are at a pretty high level among the strong sea clutter range bins. It shows that in range bins like this, the state of sea clutter is relatively stable. This means that the ionospheric disturbance is not very severe and the state of the sea clutter is more similar to HFSWR. The high correlation coefficients indicate that the iid training data is accessible in some specific range bins. But still, the influence of the ionospheric channel makes the sea clutter statistic changes unpredictable. So how to find the effective training data adaptively to do the clutter suppression in every single range bin is the key problem. The method presented in this paper is going to solve the problem in this situation.

2.2. Spatial Characteristics of Sea Clutter. To suppress the sea clutter, there are a series of methods which have been proposed. In terms of the HF surface wave radar, the mainlobe cancellation method is a powerful method for sea clutter suppression which makes use of the strong clutter spatial correlation to obtain the target-free training data. The sea clutter will be received in most beams, owing to the direction of receiving antenna and the geographical environment. The correlation is strong in most observation directions. This is the significant difference from the spatial characteristics of the target. The target can only be received in a certain direction which can be blocked by a well-designed blocking filter.

So the spatial characteristics need to be analysed if the cancellation method is going to be applied to the HFSSWR. Figure 5 shows the azimuth-Doppler map of the first-order sea clutter and a noncooperative target in the 58-range bin together. As we can see, the sea clutter occupies almost all the observation directions which are about $-40^{\circ}$ to $45^{\circ}$ and the noncooperative target only exits in a direction range of $-15^{\circ} \sim 6^{\circ}$. 


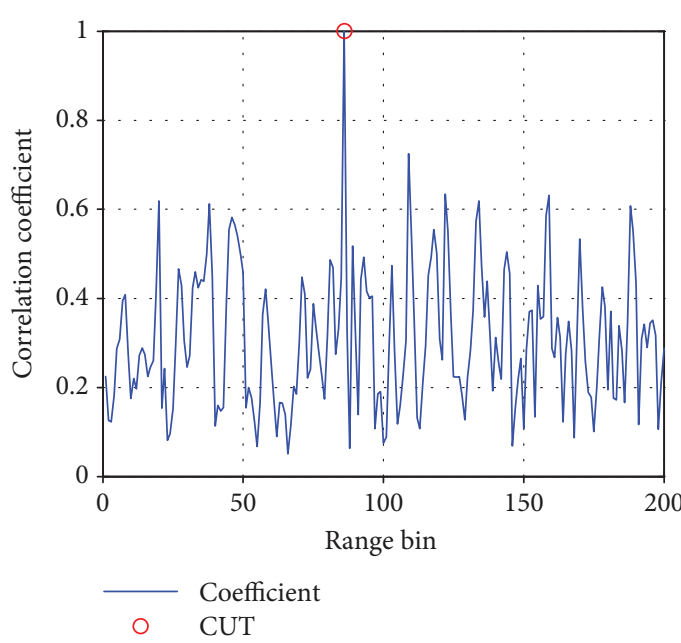

(a) Low correlation coefficient

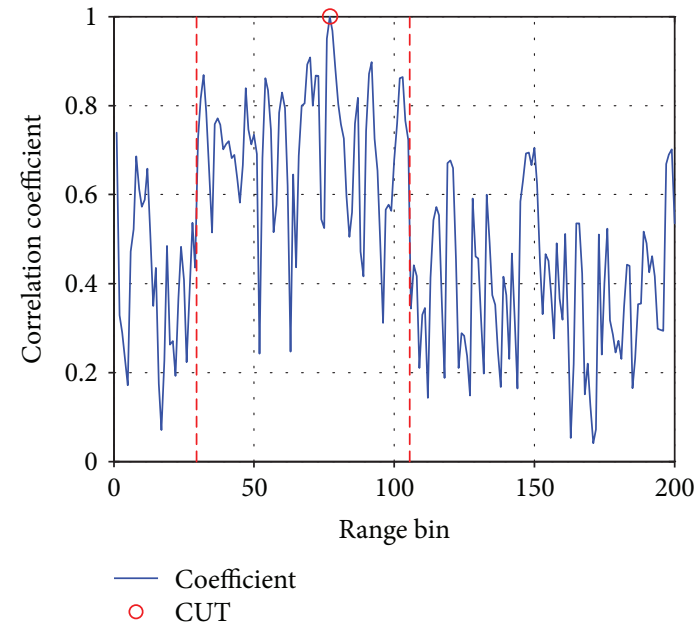

(b) High correlation coefficient

FIgURE 4: Range correlation coefficients in different situations.

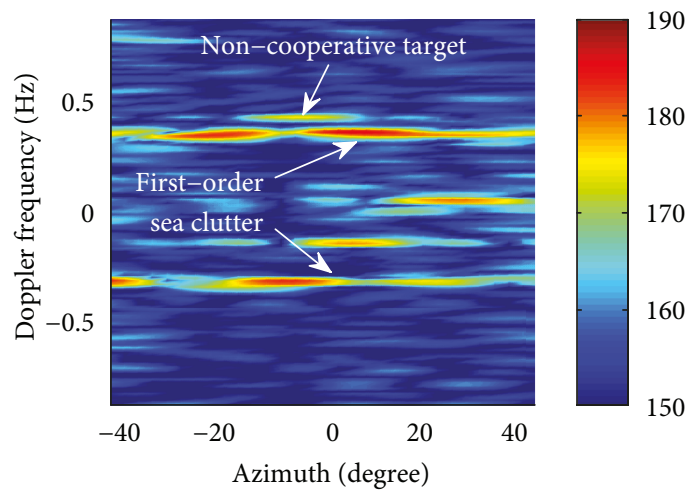

FIgURe 5: Azimuth-Doppler map of sea clutter and target.

One advantage of the main-lobe cancellation method is that it can deal with the situation where the target is submerged in the main-lobe. The method achieves the clutter cancellation by estimating the sea clutter in the main-lobe with the clutter in the side-lobe. So in order to get the accurate estimation of the main-lobe clutter, it requires the clutter data having a strong spatial correlation. The spatial correlation can be calculated by

$$
\eta_{m, k}=\frac{\left(1 / N_{f_{\mathrm{d}}}\right) \sum_{N_{f_{\mathrm{d}}}} Z_{\mathrm{r}}\left(m, f_{\mathrm{d}}\right) Z_{\mathrm{r}}^{*}\left(k, f_{\mathrm{d}}\right)}{\sqrt{\left(1 / N_{f_{\mathrm{d}}}\right)^{2} \sum_{N_{f_{\mathrm{d}}}}\left|Z_{r}\left(m, f_{\mathrm{d}}\right)\right|^{2} \sum_{N_{f_{\mathrm{d}}}}\left|Z_{\mathrm{r}}^{*}\left(k, f_{\mathrm{d}}\right)\right|^{2}}},
$$

where $N_{f_{\mathrm{d}}}$ is the number of the sample Doppler bin, $m$ is the reference beam bin, $k=1,2, \ldots, B$ is the number of whole beam bins which are going to be analysed, and $Z_{\mathrm{r}}$ is the sample data in the range bin $r$ with Doppler shift $f_{d}$.

Figure 6 gives the typical result of the first-order sea clutter spatial spectrum and corresponding correlation coefficient. Figure 7 shows a noncooperative target's spatial spectrum as the comparison. We can see that the sea clutter has a strong correlation in a wide direction scope, about 80 degrees, but the target has an obvious directionality. It should be noted that the clutter energy decreases at the azimuth range of $-45^{\circ}$ to about $-36^{\circ}$ which leads the correlation falls. The decrease in the echo's energy may be caused by an island shadowing effect in that azimuth range. And the sea clutter echo may be contaminated by the ionosphere which can also lead to inhomogeneity in HFSSWR. However, in most azimuth scopes, the correlation remains high. This means the clutter in most beams is homogeneous with the clutter in the main lobe. So we can get the conclusion that the main-lobe cancellation method is capable of suppressing the sea clutter in the background of HFSSWR theoretically for the application conditions which are satisfied based on the results of Section 2 .

\section{Improved Main-Lobe Cancellation Method}

In this section, we introduce the improved main-lobe cancellation (IMLC) method. The traditional main-lobe cancellation method is developed from the generalized side-lobe canceller (GSC) in [14]. It has been presented to suppress the ionospheric clutter and the sea clutter for ocean remote sensing by Xianrong et al. [15] and for target detection by Zhang et al. $[8,16]$. The framework of the method is shown in Figure 8.

The first step of the method is to get the target-free training data in order to estimate the clutter plus noise covariance matrix. By previous research, we found that the first-order sea clutter in HFSSWR has a strong correlation in the spatial dimension, so that we can utilize the clutter information from other directions to estimate the clutter in the looking direction. The training data used to estimate the clutter information must contain no target information to prevent the target self-elimination. The block matrix here is formed by a single notch space filter. In some situations, there are ?multiple targets in one main-lobe direction, or the training data of the sea clutter may contain not only one clutter 


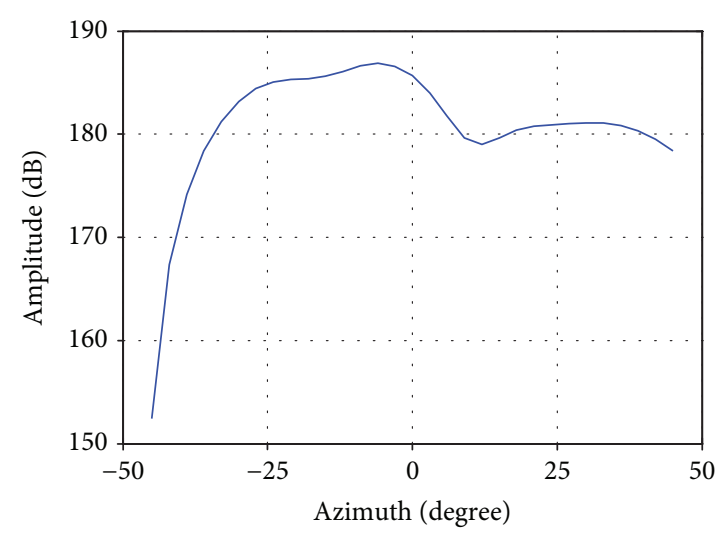

(a) Clutter spatial spectrum

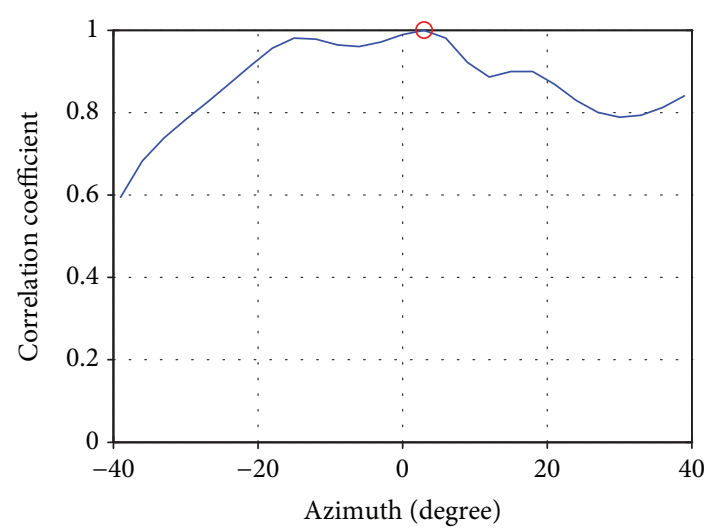

(b) Clutter spatial correlation coefficient

FIgURE 6: Clutter spatial characteristics.

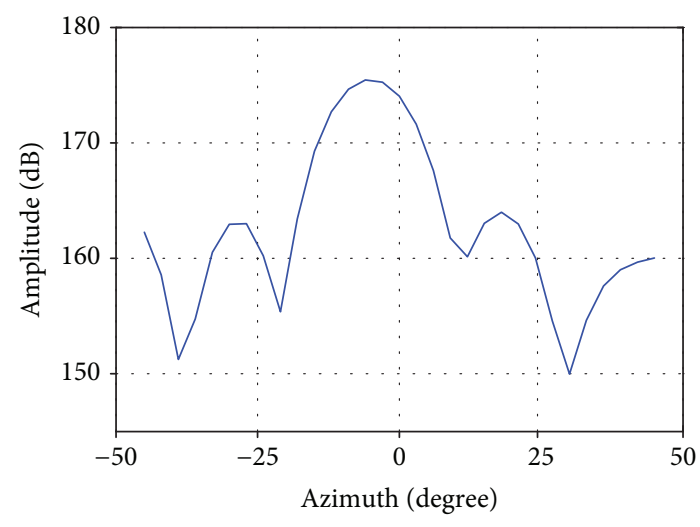

FIgURE 7: Target spatial spectrum.

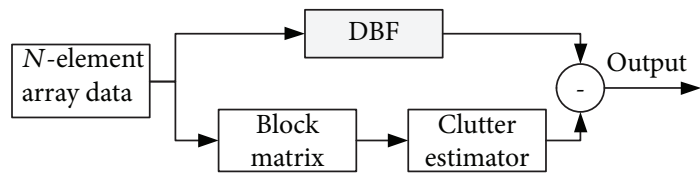

Figure 8: Framework of the MLC.

information. In this case, a large degree of freedom (DOF) is required. To ensure that the system has enough DOF, an auxiliary channel is built by way of a sliding subarray to obtain the secondary data.

In a nonstationary environment, like HFSSWR, how to select the efficient training data is a key problem in the clutter cancellation method. So we propose a training data selection strategy to improve the main-lobe cancellation method performance for the HFSSWR sea clutter suppression. The new framework of the IMLC is shown in Figure 9.

In Section 3.1, the data model of the IMLC method is introduced briefly. In Section 3.2, a single notch space filter is used to block the target signal in the main lobe. In Section 3.3, the analysis results of the amplitude-phase error are given.

3.1. Data Model. For an $N$-element uniform linear array (ULA) shown in Figure 10 which collects $M$ data snapshots, with element spacing $d$ and carrier wavelength $\lambda$, the echo data of the ith range cell can be expressed as [17]

$$
\mathbf{x}_{i}=a(i) \mathbf{s}_{\mathrm{s}-\mathrm{t}}(\theta, f)+\mathbf{c}(i)+\mathbf{n}(i),
$$

where $\mathbf{s}_{\mathrm{s}-\mathrm{t}}(\theta, f)=\mathbf{s}_{\mathrm{s}}(\theta) \cdot \mathbf{s}_{\mathrm{t}}^{\mathrm{T}}(f)$ is the target vector, $\mathbf{s}_{\mathrm{s}}(\theta)=$ $[1, \ldots, \exp (j 2 \pi(N-1) d \sin \theta / \lambda)]^{\mathrm{T}}$ is the spatial steering vector, $\mathbf{s}_{\mathrm{t}}(f)=[1, \ldots, \exp (j 2 \pi(M-1) f)]^{\mathrm{T}}$ denotes the temporal steering vector, $a(i)$ is the target amplitude, $\mathbf{c}(i)$ denotes the clutter, and $\mathbf{n}(i)$ denotes the noise which is nondirectional. And $(\cdot)^{T}$ denotes transpose.

The covariance matrix of the cell under test can be written as

$$
\mathbf{R}_{\mathrm{CUT}}=E\left[\mathbf{x}_{\mathrm{CUT}} \mathbf{x}_{\mathrm{CUT}}^{\mathrm{H}}\right]=\sigma_{\mathrm{t}}^{2} \mathbf{s}_{\mathrm{s}-\mathrm{t}} \mathbf{s}_{\mathrm{s}-\mathrm{t}}^{\mathrm{H}}+\mathbf{R}_{\mathrm{u}},
$$

where $\sigma_{\mathrm{t}}^{2}=|\alpha(i)|^{2}$ is the power of the target, $\mathbf{R}_{\mathrm{u}}=\mathbf{R}_{c}+\mathbf{R}_{n}$ $=E\left[\mathbf{c}_{\mathrm{CUT}} \boldsymbol{c}_{\mathrm{CUT}}{ }^{\mathrm{H}}\right]+\sigma_{\mathrm{n}}^{2} \mathbf{I}$, where $\sigma_{\mathrm{n}}^{2}$ is the noise power and $\mathbf{I}$ denotes the identity matrix, and $(\cdot)^{\mathrm{H}}$ denotes the Hermitian transpose.

In practical applications, $\mathbf{R}_{\mathrm{u}}$ is unknown and has to be estimated from the training data around the CUT.

$$
\widehat{\mathbf{R}}_{\mathrm{u}}=\frac{1}{K} \sum_{k \in \Gamma} \mathbf{x}_{k} \mathbf{x}_{k}^{H},
$$

where $K$ is the total number of target-free training data, and $\Gamma$ is the set of the training samples in the range domain. And the aim of the proposed method is to select the most valid training data.

3.2. Single Notch Space Filter. In order to block the target information, a single notch space filter (SNSF) is proposed as the block matrix. The least P-norm FIR filter is chosen for its side-lobe response which is approximately equal. This means that the clutter information in the side-lobe can be kept maximally. The beam null steering points to the same direction as the main-lobe so that only the clutter and the noise in the side-lobe will be remained. The response is 


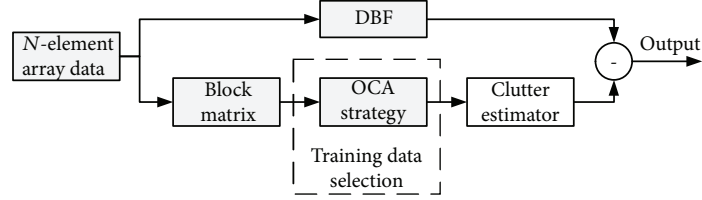

FIGURE 9: Framework of the IMLC.

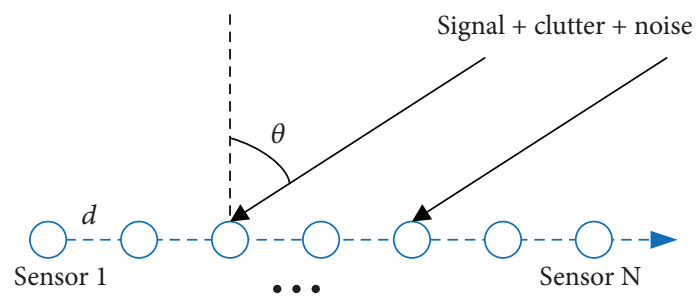

FIGURE 10: Uniform linear array.

shown in Figure 11 with $N=16$ and $L=10$, the DOF is 7 , and the main-lobe direction is $-15^{\circ}$.

In order to fully block the target component, the filter needs to be well designed based on two principles. Firstly, the depth of the filter should be deeper than the target power. Secondly, the width of the filter should be wider than the main lobe. In this case, we can assume that after filtering, there are no main-lobe components left in the secondary data. Here, they are antithetic conditions and should be carefully designed to ensure the filtering effect.

3.3. Analysis of Amplitude-Phase Error. There are some limits of the main-lobe cancellation method. As discussed in the above section, the single notch filter needs to be carefully designed based on the two principles. And the degree of freedom is limited by the practical system as well. But one of the most severe problems is the amplitude-phase error in the receiving antennas.

The amplitude error will change the level of the side-lobe response, and the phase error will cause the decrease of the notch depth. In some extreme situations, the notch may even disappear due to the heavy phase error, so that the target information will be remained in the training data which will lead to target self-elimination. The compensation of the amplitude-phase error is essential before the signal processing. Here, we give the analysis of the amplitude-phase errors' impact on the single notch filter to guide the compensation processing.

First, we give the simulation result of the phase error's impact. The phase errors are set to be uniformly distributed pseudorandom numbers in different scopes to represent the different error levels. Figure 12 shows the response of the single notch filter with different phase error levels. The red circle line is the ideal response with no phase error. The black square line is the response with the phase error in the $[-0.5$ $\nabla 0.5]$ degree scope. The blue asterisk line is the response with the phase error in the $\left[\begin{array}{ll}-1 & 1\end{array}\right]$ degree scope while the pink diamond line is that in $\left[\begin{array}{ll}-2 & 2\end{array}\right]$. We can see that with the phase error increase, the response becomes worse. The depth of the notch reduces, and the side-lobe responses are not equal any more. This will lead the target component to

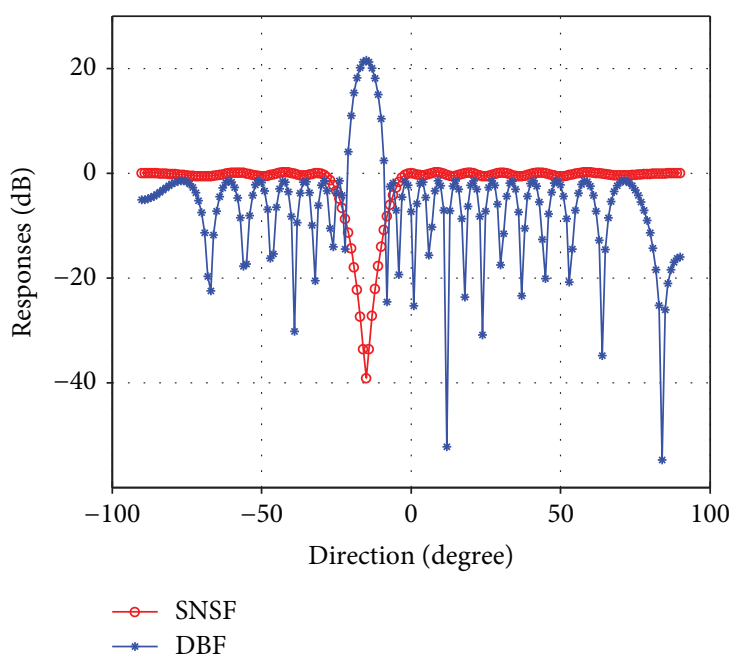

Figure 11: Response of the SNSF.

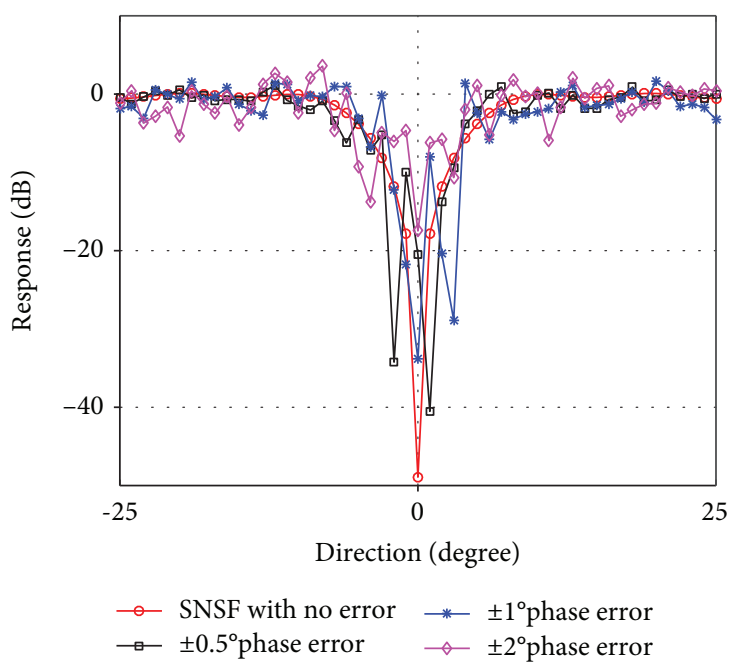

FIGURE 12: Response of SNSF with phase errors.

remain in the training data and affect the validity of the clutter suppression method.

Then, we give the simulation result of the amplitude error's impact. The amplitude errors are also set in different scopes. The impact of the amplitude errors on the single notch filter response is shown in Figure 13. The ideal response with no amplitude error is still shown with the red circle line. And the black square line, the blue asterisk line, and the pink diamond line represent the amplitude errors in $\left[\begin{array}{ll}-1 & 1\end{array}\right] \mathrm{dB},\left[\begin{array}{ll}-2 & 2\end{array}\right] \mathrm{dB}$, and $\left[\begin{array}{ll}-5 & 5\end{array}\right] \mathrm{dB}$ scopes, respectively. It can be seen from the simulation result that the impact of amplitude errors is on the inequality of the side-lobe response. It hardly affects the depth and direction of the notch.

In conclusion, the amplitude-phase error is a key problem which will affect the clutter suppression result. So it is necessary to do the compensation processing before signal processing to ensure the amplitude-phase consistency. In 


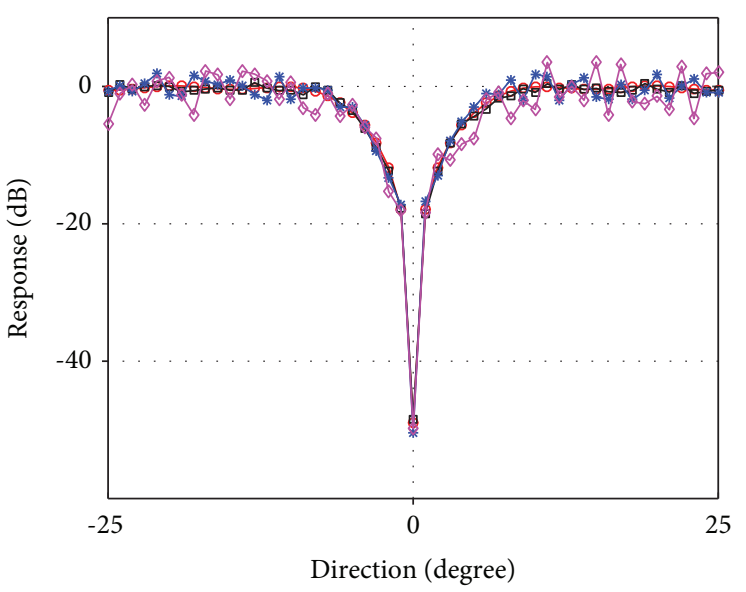

$\rightarrow$ SNSF with no error $\quad-* \pm 2$ nbsp dB amplitude error $\rightarrow \pm 1$ nbsp dB amplitude error $\multimap \pm 5$ nbsp dB amplitude error

FIGURE 13: Response of SNSF with amplitude errors.

the practical system, the allowed margin of the amplitude error is $\pm 2 \mathrm{~dB}$ while the phase error is limited at $\pm 1^{\circ}$.

\section{Improved Training Data Selection}

Generally, the STAP method requires at least twice the DOF samples of training data to obtain an accurate covariance matrix estimation [18]. But in the practical HFSSWR system, it is difficult to provide enough training data which is independent and identically distributed with the CUT. The nonstationary property of the ionosphere causes the fluctuation of clutter characteristics in range bins, discussed in Section 2.1. This makes the conventional main-lobe cancellation method become invalid in the heterogeneous environment. In this section, the improved training data selection method is introduced based on an optimized correlation analysis strategy (OCA) using spatial eigenvectors to choose the efficient secondary data. This strategy tells how to define the parameter $k$ mentioned in Section 3.1. The aim of the method is to find the strong correlation data to be selected as the training data that is more consistent with the clutter statistic distribution, under the premise of limited training samples. And in HFSSWR, the first-order sea clutter will broaden due to the ionospheric contamination and bistatic system configuration. So in order to fully express the clutter information, the localized processing region (LPR) has been introduced into the IMLC. And the simulation results of the broadening value of the first-order sea clutter are presented to improve the selection of the LPR.

In Section 4.1, the proposed optimized correlation analysis strategy is introduced. During the OCA processing, the LPR is used to better represent the clutter information. So in Section 4.2, how to define the range of the LPR is discussed.

4.1. Optimized Correlation Analysis Strategy. In the case of HFSSWR, the first-order sea clutter data in the range domain change significantly due to the nonstationary properties of the ionosphere based on the analysis results in Section 2.1.
So the adjacent range bins cannot provide the MLC required iid data. The echo data in one range cell often consists of different components of target, sea clutter, and noise. The output of the SNSF in Section 3.2 contains no target information, and the power of the background noise is weaker than the strong sea clutter. So the strong eigenvalues in one LPR represents the sea clutter characteristic in the Bragg peak region. In ideal situations in which the sea clutter is pure and no other interference exists, there will be only one dominant eigenvalue corresponding to the sea clutter. But due to the ionospheric contamination, the first-order sea clutter is not pure; there may be more than one dominant eigenvalue. The optimized correlation analysis strategy proposed here is to select the effective neighbouring range cells to be the training data. The strategy utilizes the eigenvectors which have greater contribution to calculate the correlation coefficient with the CUT and then select the range cell data whose coefficient is greater than the threshold to be the training data.

The OCA strategy is operated as follows:

(1) Select a two-dimensional angle-Doppler localized processing region (LPR) for one-range cells and convert to a column vector $X_{A-D}=\left[x_{1}, x_{2}, \ldots, x_{\mathrm{T}}\right]^{\mathrm{T}}$, where $T=p \times q$ is the size of the LPR, $p$ is the number of the angle domain data, and $q$ is the number of the Doppler domain data

(2) Calculate its space-time covariance matrix $R$

$$
R=X_{A D} \cdot X_{A D}^{\mathrm{T}}
$$

(3) Do the eigenvalue decomposition (EVD) of $R$, and get $T$ eigenvalues $\lambda=\left[\lambda_{1}, \lambda_{2}, \ldots, \lambda_{\mathrm{T}}\right]^{\mathrm{T}}$ and the corresponding angle-Doppler joint domain normalized eigenvectors $\xi=\left[\xi_{1}, \xi_{2}, \ldots, \xi_{\mathrm{T}}\right]^{\mathrm{T}}$

(4) Calculate the contribution value of each eigenvector and find the eigenvector $\varsigma$ with the maximum value to represent the clutter characteristic in the current range cell by the following equation $\lambda_{i} \xi_{i}^{\mathrm{H}}$ $X_{A-D}, i=1,2, \ldots, T$

(5) Do the process on $K$ range cells based on steps (1) to (4) and obtain the $K$ normalized eigenvectors $\varsigma=\left[\begin{array}{llll}\varsigma_{1} & \varsigma_{2} & \ldots & \varsigma_{K}\end{array}\right]^{\mathrm{T}}$

(6) Calculate the correlation coefficient $\rho_{i j}$ between the eigenvector $\varsigma_{i}$ for the range cells under test and other range cells

$$
\rho_{i j}=\varsigma_{i}^{\mathrm{H}} \varsigma_{j}, \quad j=1,2, \ldots, K
$$

(7) Select the effective training data $X_{c}(f)=$ $\left[\begin{array}{llll}x_{r, 1} & x_{r, 2} & \ldots & x_{r, k}\end{array}\right]$ whose correlation coefficient is continuously greater than the threshold $\eta$ from the range cell around the cell under test 


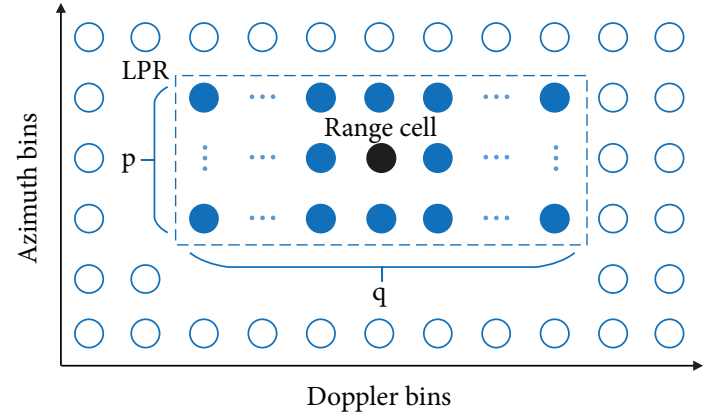

FIGURE 14: Size of the LPR

4.2. Localized Processing Region Discussion. The broadening first-order sea clutter can cover a few Doppler bins, so in order to extract the accurate sea clutter information, a localized processing region data is taken into account for the first step in Section 4.1. The size of the LPR is shown in Figure 14.

The size of the LPR is worthy to be discussed. In the azimuth dimension, based on the analysis results in Section 2.2, the first-order sea clutter has a strong spatial correlation in the azimuth dimension. The correlation is in a high level in a few azimuth bins. This means that spatial clutter information can be better expressed utilized a few azimuth bins. But the more LPR data are used, the heavier the computational cost will be. So in practice, we take $p=3$. In the Doppler dimension, the first-order sea clutter will broaden in hybrid sky-surface wave mode owing to the bistatic radar mode and ionospheric phase contamination. So the size of LPR in Doppler bins should be calculated by the Bragg frequency range.

In [6], the Bragg frequency range of the broadening first-order sea clutter is calculated

$$
\begin{aligned}
& {\left[ \pm 0.102 \times 10^{-3} \sqrt{f_{0} \cdot \cos \left(\beta_{\mathrm{H}^{\prime}} / 2\right)}+f_{\text {ion_min }},\right.} \\
& \left. \pm 0.102 \times 10^{-3} \sqrt{f_{0} \cdot \cos \left(\beta_{\mathrm{H}^{\prime}} / 2\right)}+f_{\text {ion_max }}\right]
\end{aligned}
$$

where $f_{0}$ is the carrier frequency, $\beta_{\mathrm{H}^{\prime}}$ is the modified bistatic angle, and $f_{\text {ion }}$ is the Doppler shift caused by the ionosphere.

Figure 15 gives the simulation result of the broadening range of the first-order sea clutter. The comparison has been made between the measured data and the theoretical calculation. It is the result of the No. 53 range bin, and main-lobe direction is $0^{\circ}$. The carrier frequency is $10.91 \mathrm{MHz}$, and the modified bistatic angle is $14.41^{\circ}$. From Figure 15, we can see that the theoretical value matches the measured data very well. And the parameter $q$ can be calculated by dividing the range of Equation (8) by Doppler resolution, which is $0.013 \mathrm{~Hz}$. We can get $q \approx 7$ in this situation.

\section{Experimental Results}

The measured data is used to verify the effectiveness of the IMLC method. The results are compared with the DBF method and traditional main-lobe cancellation method.

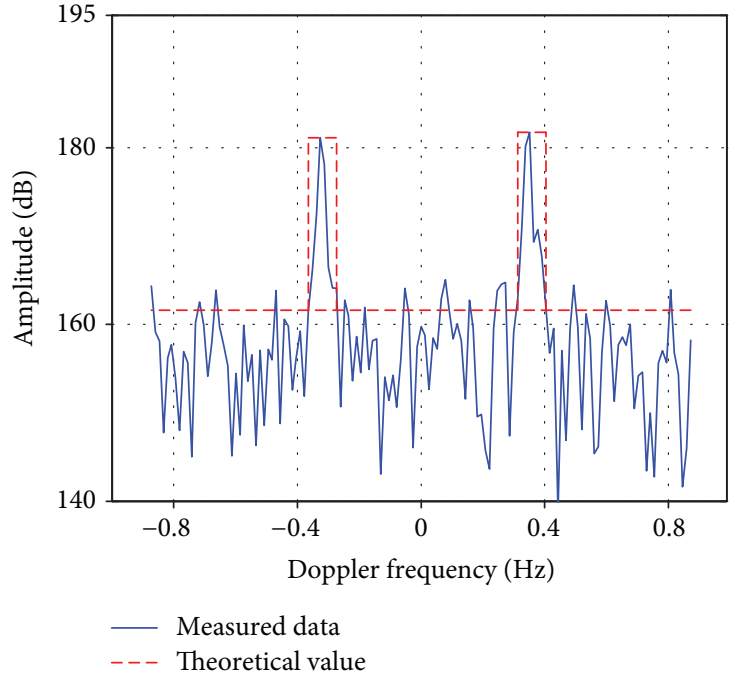

Figure 15: Comparison of the broadening value of the first-order sea clutter.

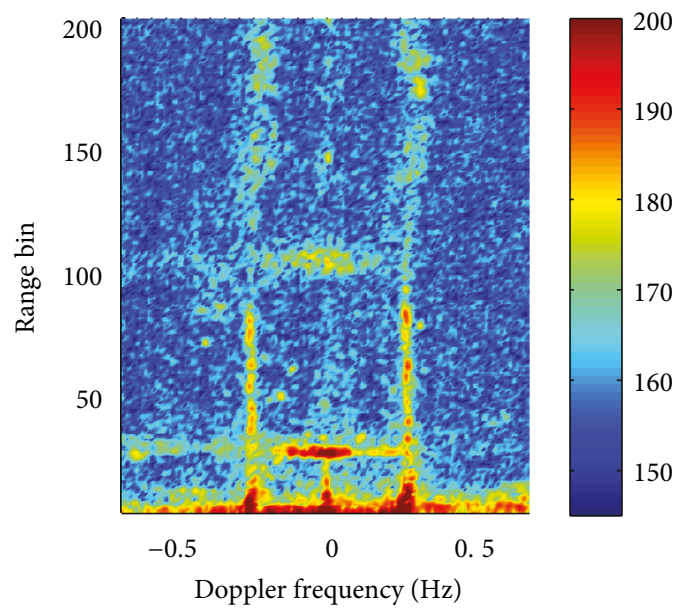

FIGURE 16: Range-Doppler maps by the DBF method.

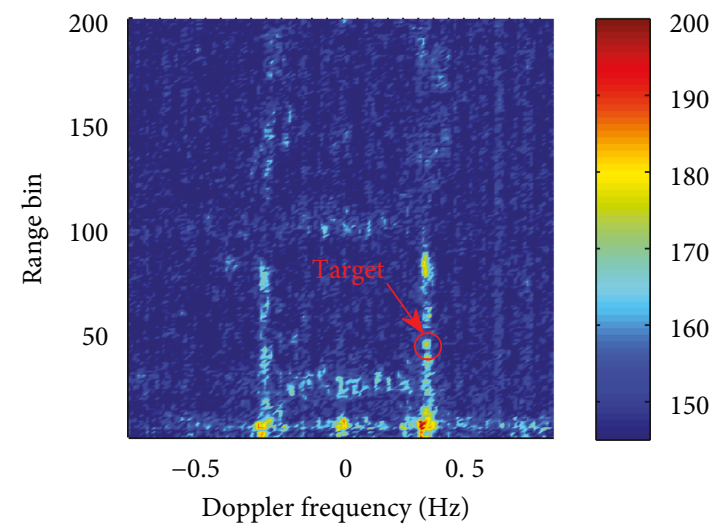

FIGURE 17: Range-Doppler maps by the IMLC method.

The measured data is collected from a 16-element ULA with a 10-meter element interval. The transmitted signal is the phase-coded signal with a carrier frequency of $10.91 \mathrm{MHz}$, 


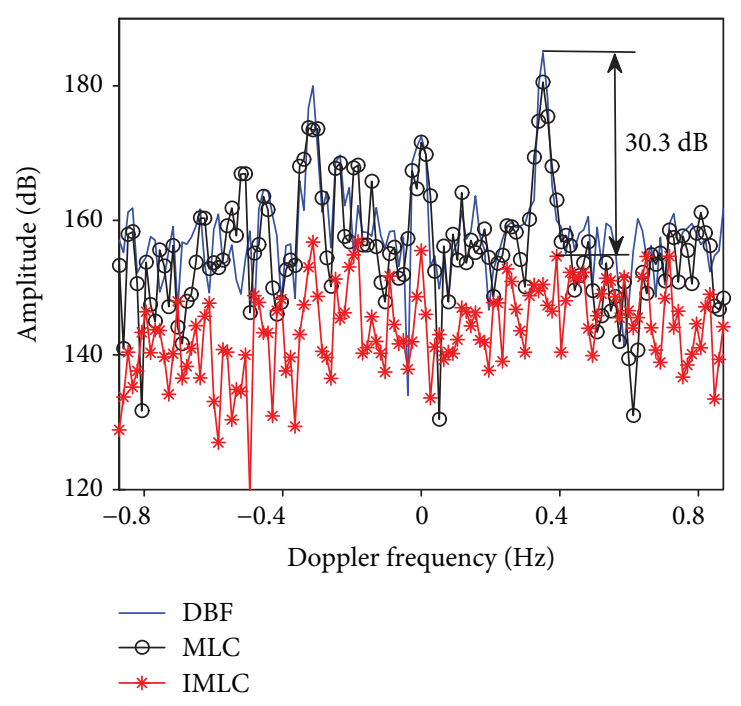

(a) Clutter suppression result

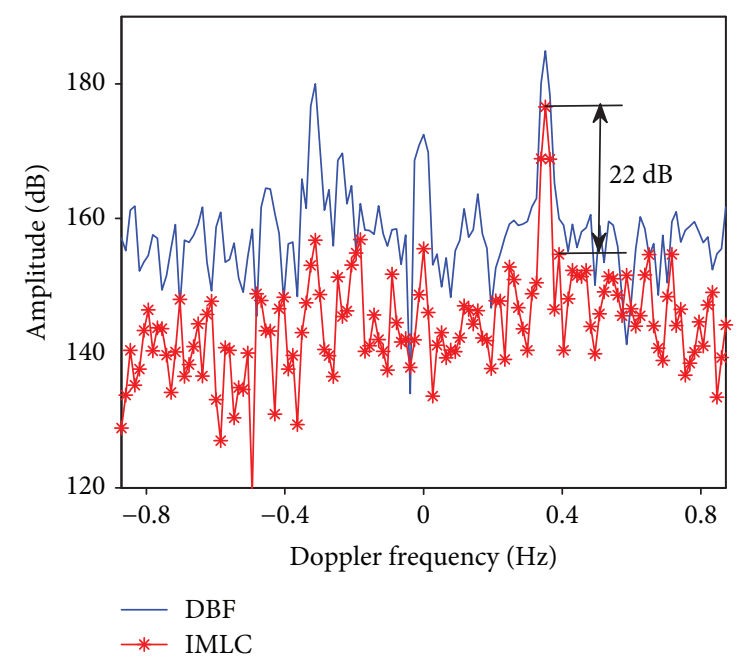

(b) Injecting target suppression result

FIGURE 18: Suppression results for clutter and clutter plus target.

bandwidth $40 \mathrm{kHz}$, and pulse repetition frequency of $66.67 \mathrm{~Hz}$.

5.1. Measured Data Plus a Simulated Target. Firstly, we inject a simulated target at no. 45 range cells, $0.3516 \mathrm{~Hz}$ Doppler frequency, and degree of $0^{\circ}$. In this case, the modified bistatic angle is $14.6248^{\circ}$. At this Doppler frequency, the target is completely submerged by the broadening first-order sea clutter which makes it impossible to be detected.

Figures 16 and 17 show the range-Doppler plots of the DBF method and IMLC method, respectively. By comparison of the two figures, it can be seen that the broadening first-order sea clutter has been largely suppressed and the target can been seen, although it is not isolated due to the clutter residual. This situation which is the most severe situation for target detection shows that the IMLC is a very powerful method to suppress the clutter and protects the target information. Also, it can be seen that some suspected targets have been removed together with the clutter. This can be explained that the azimuths of the suspected targets are largely mismatched with the main lobe at this direction. If the main lobe turns to the azimuths of these targets, they will be protected by the SNSF.

Figure 18 shows the Doppler profile results of no. 45 range cells obtained by 3 different methods. The DBF method and the MLC method are taken here as comparisons. Figure 18(a) is the suppression result with no target injected. It shows clearly that due to the effect of the ionospheric propagation channel, the traditional MLC method has nearly no effect on sea clutter. The IMLC method can suppress the sea clutter to a large extent; it is weakened by the maximum of $30.3 \mathrm{~dB}$ for the positive first-order sea clutter and about $23 \mathrm{~dB}$ for the negative first-order sea clutter. Figure 18(b) shows that IMLC can protect the target while suppressing the clutter and the improvement of SCNR is up to $22 \mathrm{~dB}$. It makes a great advantage for detecting the low velocity vessels which are submerged by the sea clutter in the main lobe.

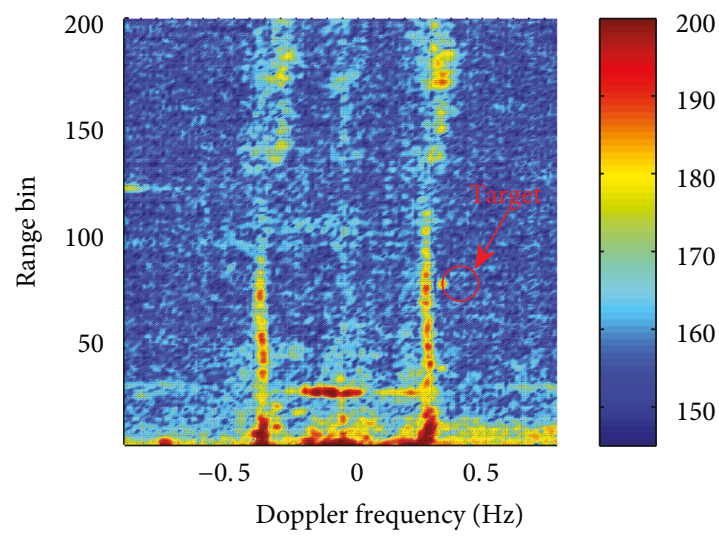

Figure 19: Range-Doppler map by the DBF method.

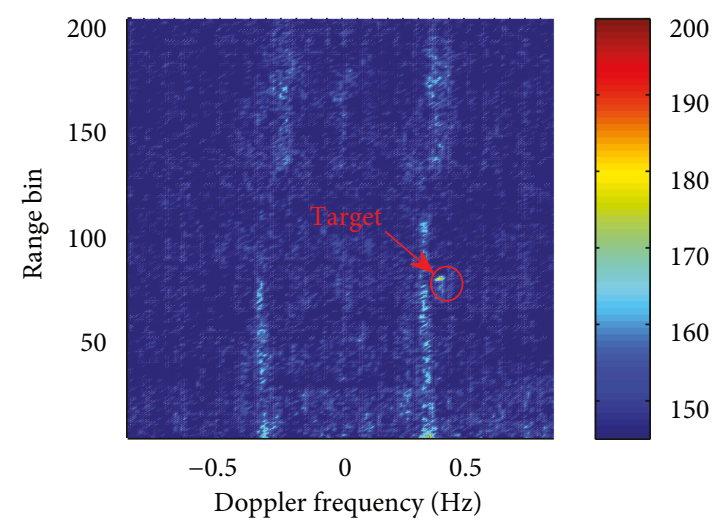

FIGURE 20: Range-Doppler map by the IMLC method.

5.2. Measured Data with a Noncooperative Target. Then, we found a noncooperative target in the measured data to show the effectiveness of the proposed method in practical 


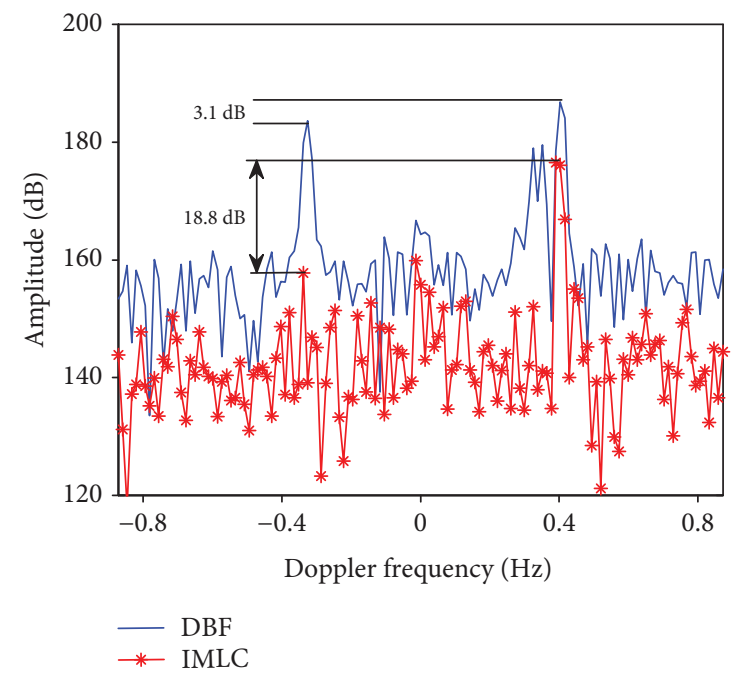

FIGURE 21: Suppression result for the noncooperative target.

situations. The noncooperative target is at the no. 76 range cell, $0.4036 \mathrm{~Hz}$, and the target direction is $-15^{\circ}$.

Figures 19 and 20 show the comparison of the DBF method and the IMLC method. Figure 20 shows that the IMLC method can suppress the broadening first-order sea clutter effectively and protect the target in the main lobe. The method is proved to be a useful way to suppress the sea clutter in the background of the HFSSWR. The method brings a great benefit on SCNR improvement.

To show the cancellation result more clearly, the Doppler profile of the target range cell is shown in Figure 21. Compared with the DBF method, it can be seen that the sea clutter has been greatly suppressed. The negative Bragg peak has been weakened by $26 \mathrm{~dB}$, and the positive Bragg peak result is $27 \mathrm{~dB}$. And we can see that although the target suffers a little SNR loss due to the limitation of the training data, SCNR has been improved from $3.1 \mathrm{~dB}$ to $18.8 \mathrm{~dB}$. This will benefit the following target detection processing.

\section{Conclusions}

In this paper, an effective clutter cancellation algorithm, IMLC, is proposed for broadening first-order sea clutter suppression. It applies the main-lobe cancellation method to the clutter suppression with the background of HFSSWR. More detailed problems have been discussed, and new simulation and experimental results have been given on the basis of the conference paper. The range and spatial characteristics of the first-order sea clutter in HFSSWR are first discussed to guide the suppression algorithm design. The analysis results show that the influence of the ionospheric channel makes the sea clutter change unpredictably in the range domain. In some situations, the state of the first-order sea clutter is not stable at even the adjacent range bins. And in the spatial domain, the first-order sea clutter shows the high correlation property in most observation directions. This forms the theoretic foundation of the MLC method. In order to solve the degradation of the traditional MLC method caused by the ionospheric nonstationary property, we improve the method to select more efficient training data. A single notch space filter is chosen as the block matrix, and the influences of the amplitude-phase error have been shown. An improved training data selection method based on spatial eigenvector correlation analysis, which we call optimized correlation analysis strategy, is proposed. The broadening Bragg frequency of the first-order sea clutter in HFSSWR is taken into consideration. The experimental results, utilizing the simulated target as well as the noncooperative target, show the effectiveness of the algorithm which can suppress the first-order sea clutter and improve the SCNR of the target. For further application, the IMLC algorithm can be used in other situations facing a similar challenge.

\section{Data Availability}

The measured data used to support the findings of this study have not been made available because of data sensitivity.

\section{Conflicts of Interest}

The authors declare that there is no conflict of interest regarding the publication of this paper.

\section{Acknowledgments}

This work has been supported by the National Natural Science Foundation of China under grants 61171182 and 61032011 and the Fundamental Research Funds for the Central Universities under grants HIT.MKSTISP.2016 13 and 26. The authors are grateful to the Institute of Electronic and Information Technology, Harbin Institute of Technology, who provided the HFSSWR data and those who helped during the writing of this paper.

\section{References}

[1] Y. Wei, P. Tong, R. Xu, and L. Yu, "Experimental analysis of a HF hybrid sky-surface wave radar," IEEE Aerospace and Electronic Systems Magazine, vol. 33, no. 3, pp. 32-40, 2018.

[2] R. J. Riddolls, "Limits on the detection of low-Doppler targets by a high frequency hybrid sky-surface wave radar system," in 2008 IEEE Radar Conference, pp. 1-4, Rome, 2008.

[3] I. S. Turgenev and P. A. Melyanovcky, "Bistatic HF radar for oceanography applications with the use of both ground and space waves," Telecommunications and Radio Engineering, vol. 51, no. 2-3, pp. 73-80, 1997.

[4] Z. Zhao, X. Wan, D. Zhang, and F. Cheng, "An experimental study of HF passive bistatic radar via hybrid sky-surface wave mode," IEEE Transactions on Antennas and Propagation, vol. 61, no. 1, pp. 415-424, 2013.

[5] L. Ya-jun, W. Yin-sheng, X. Rong-qing, and S. Chao, "Simulation analysis and experimentation study on sea clutter spectrum for high-frequency hybrid sky-surface wave propagation mode," IET Radar, Sonar \& Navigation, vol. 8, no. 8, pp. 917930, 2014.

[6] L. Ya-jun, W. Yin-sheng, Z. Yong-peng, G. Ru-jiang, W. Zhuoqun, and X. Rong-qing, "Analysis and simulation for broadening first-order sea clutter spectrum in high frequency hybrid sky-surface wave propagation mode," IET Radar, Sonar \& Navigation, vol. 9, no. 6, pp. 609-621, 2015. 
[7] Y. Li, Y. Wei, Y. Zhu, Z. Wang, and R. Xu, "Cascaded method for ionospheric decontamination and sea clutter suppression for high-frequency hybrid sky-surface wave radar," IET Signal Processing, vol. 9, no. 7, pp. 562-571, 2015.

[8] X. Zhang, Q. Yang, D. Yao, and W. Deng, "Main-lobe cancellation of the space spread clutter for target detection in HFSWR," IEEE Journal of Selected Topics in Signal Processing, vol. 9, no. 8, pp. 1632-1638, 2015.

[9] R. S. Adve, T. B. Hale, and M. C. Wicks, "Practical joint domain localised adaptive processing in homogeneous and nonhomogeneous environments," IEE Proceedings - Radar, Sonar and Navigation, vol. 147, no. 2, pp. 57-65, 2000.

[10] I. S. Reed, J. D. Mallett, and L. E. Brennan, "Rapid convergence rate in adaptive arrays," IEEE Transactions on Aerospace and Electronic Systems, vol. AES-10, no. 6, pp. 853-863, 1974.

[11] B. A. Johnson and Y. I. Abramovich, "Low training volume adaptive processing in HF skywave radar," in 2008 International Conference on Radar, pp. 616-621, Adelaide, 2008.

[12] P. Tong, Y. S. Wei, and R. Q. Xu, "Single dataset method for spread-Doppler clutter suppression in HF hybrid sky-surface wave radar," Electronics Letters, vol. 53, no. 4, pp. 277-279, 2017.

[13] J. Zhang, W. Deng, X. Zhang, and Q. Yang, "Improved mainlobe cancellation method for space spread clutter suppression in HFSSWR," in 2018 IEEE Radar Conference (RadarConf18), pp. 197-201, Oklahoma City, 2018.

[14] K. Buckley and L. Griffiths, "An adaptive generalized sidelobe canceller with derivative constraints," IEEE Transactions on Antennas and Propagation, vol. 34, no. 3, pp. 311-319, 1986.

[15] W. Xianrong, K. Hengyu, and W. Biyang, "Adaptive ionospheric clutter suppression based on subarrays in monostatic HF surface wave radar," IEE Proceedings - Radar, Sonar and Navigation, vol. 152, no. 2, pp. 89-96, 2005.

[16] X. Zhang, D. Yao, Q. Yang, Y. N. Dong, and W. B. Deng, "Knowledge-based generalized side-lobe canceller for ionospheric clutter suppression in HFSWR," Remote Sensing, vol. 10, no. 2, p. 13, 2018.

[17] E. Aboutanios and B. Mulgrew, "A STAP algorithm for radar target detection in heterogeneous environments," in IEEE/SP 13th Workshop on Statistical Signal Processing, vol. 2005, pp. 966-971, Bordeaux, 2005.

[18] W. L. Melvin, “A STAP overview," IEEE Aerospace and Electronic Systems Magazine, vol. 19, no. 1, pp. 19-35, 2004. 


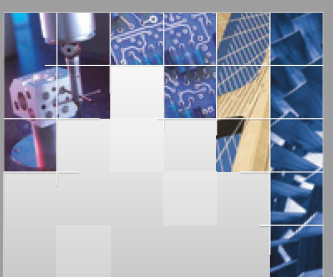

\section{Enfincering}
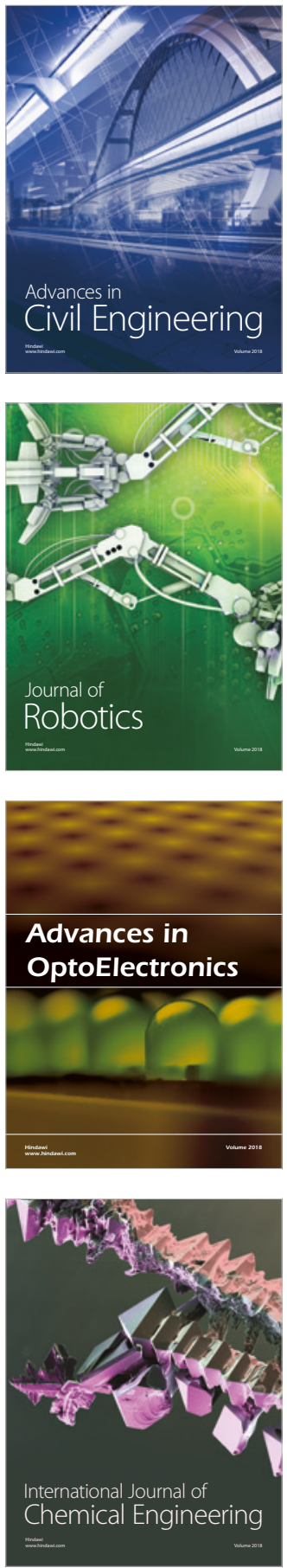

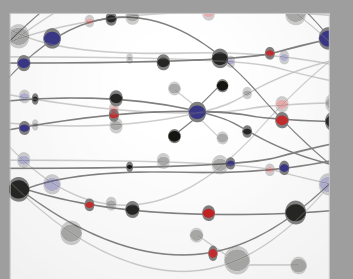

\section{Rotating \\ Machinery}

The Scientific World Journal

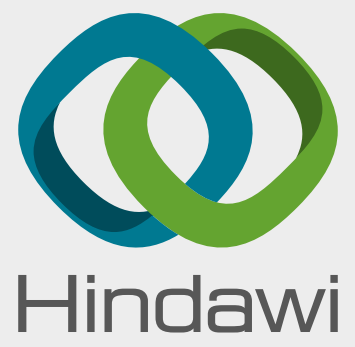

Submit your manuscripts at

www.hindawi.com
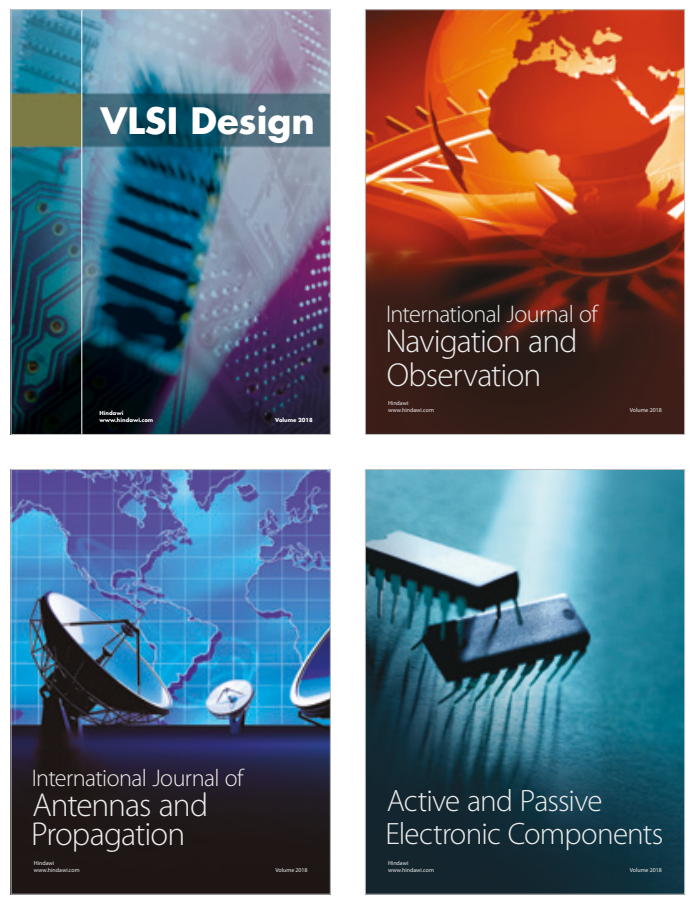
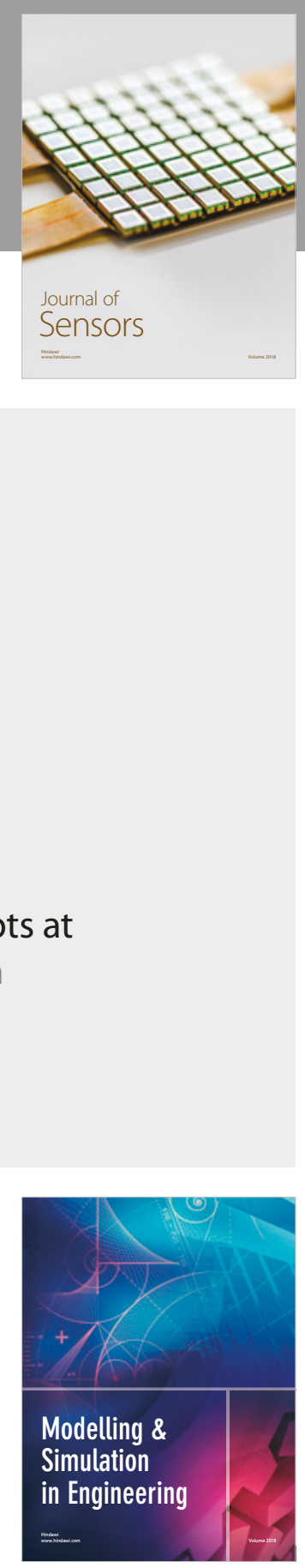

\section{Advances \\ Multimedia}
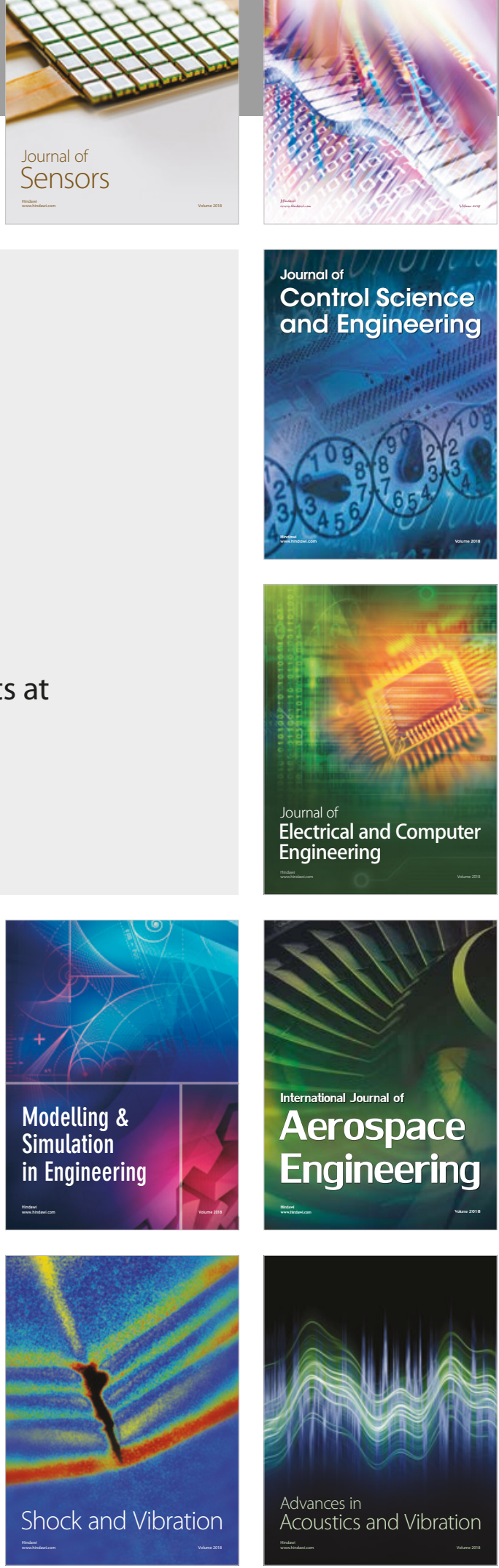